\title{
Tess Learns to Be Professional
}

"Wouldn't it be better for everyone?"

he said. But I would not lie on a slightly

stinking stone heavy with its own bowels.

He slapped me again and again

to give me no time for bewilderment, that decadent emotion.

No one cared. Birds had their sky, and the stones stayed in their posy ring, cloaked to their bald cheeks in shadow.

Even I wasn't let to care. I asked his pardon for crying, and he forgave me with the gesture of a healthy prince.

The standing stones nodded their weight like heads approving the end of soul and the birth of eyes of lead. 